\title{
Uniform standards do not apply to readmission following coronary artery bypass surgery: A multi-institutional study
}

\author{
Robert Lancey, MD, ${ }^{a}$ Paul Kurlansky, MD, ${ }^{\mathrm{b}}$ Michael Argenziano, MD, ${ }^{\mathrm{b}}$ Michael Coady, MD, ${ }^{\mathrm{c}}$ \\ Robert Dunton, MD, ${ }^{\mathrm{d}}$ James Greelish, MD, ${ }^{\mathrm{e}}$ Edward Nast, MD ${ }^{\mathrm{f}}$ Samuel Gwin Robbins, MD, ${ }^{\mathrm{g}}$ \\ Melissa Scribani, MPH, ${ }^{\mathrm{h}}$ Judy Tingley, MPH, RN, ${ }^{\mathrm{b}}$ Timothy Williams, MD, ${ }^{\mathrm{i}}$ Alex Zapolansky, MD, ${ }^{\mathrm{j}}$ and \\ Craig Smith, MD ${ }^{\mathrm{b}}$
}

Objectives: Reducing hospital readmissions is a national priority, with coronary artery bypass graft (CABG) surgery slated for upcoming reimbursement decisions. Clear understanding of the elements associated with readmissions is essential for developing a coherent prevention strategy. Patterns of readmission vary considerably based on diagnosis. We therefore sought to clarify the factors most clearly associated with 30-day readmission following CABG surgery in an academically affiliated community hospital network.

\begin{abstract}
Methods: All patients undergoing isolated CABG in an 11-hospital network from 2007 to 2011 were entered into a Society of Thoracic Surgeons (STS) compliant registry that tracks hospital readmission within 30 days of surgery. Data were split at random into training and validation groups that were used to create and validate a logistic regression model of pre-, intra-, and postoperative factors associated with readmission. Subanalyses included development of logistic models predicting readmission for the 2 largest institutions individually, and relatedness of readmission to CABG procedure.
\end{abstract}

Results: The readmission rate for the entire 4861 patient group was $9.2 \%$ and varied between hospitals from $6.1 \%$ to $18.0 \%$. Factors associated with readmission were moderate chronic obstructed pulmonary disease (odds ratio [OR], $1.81 ; 95 \%$ confidence interval $[\mathrm{CI}], 1.04-3.14 ; P=.036)$, cerebrovascular disease $(\mathrm{OR}, 1.56 ; 95 \% \mathrm{CI}, 1.09-2.24$; $P=.016)$, diabetes (OR, 1.44; 95\% CI, 1.08-1.93; $P=.014)$, congestive heart failure (OR, $2.12 ; 95 \% \mathrm{CI}$, 1.23-3.66; $P=.007)$, intra-aortic balloon pump (OR, $0.40 ; 95 \% \mathrm{CI}, 0.19-0.83 ; P=.015)$, and use of blood products (OR, 1.76; 95\% CI, 1.31-2.37; $P=.0002)$. Although the $c$ statistic for the training model $(\mathrm{n}=2341)$ was 0.643 , when applied to the validation dataset $(\mathrm{n}=2520)$ the area under the receiver operating curve was reduced to 0.57 . Separate analyses of factors for the 2 largest hospitals revealed marked differences, with only body mass index (OR, 1.08; 95\% CI, 1.04-1.12; $P=.0001)$ significantly associated with readmission at 1 hospital, and discharge to extended care (OR, $2.11 ; 95 \%$ CI, $1.02-4.33 ; P=.043)$ and renal failure (OR, $2.64 ; 95 \% \mathrm{CI}, 1.21-5.76 ; P=.0149)$ significant at the other hospital. Most readmissions $(60.8 \%)$ occurred within 10 days of discharge. Nearly one-third $(31.3 \%)$ were categorized as unlikely to be CABG-related. The mean number of days from surgery to readmission was less for readmissions clearly related to CABG (15.5 \pm 6.4 days), compared with those unlikely to be CABG-related (17.4 \pm 7.0 days) $(P=.05)$.

Conclusions: Analysis of CABG readmission data from a network of community hospitals that vary in size and patient demographic characteristics suggests that there are many nonclinical factors influencing readmission; readmission rates and associated risk factors may vary considerably between centers; earlier readmissions are more likely to be procedure-related than patient-related; and therefore, considerable caution should be exercised in attempting to apply uniform standards or strategies to address post-CABG readmission. (J Thorac Cardiovasc Surg 2015;149:850-7)

From the Maryview Medical Center, ${ }^{a}$ Portsmouth, Va; Columbia University, ${ }^{\mathrm{b}}$ New York, NY; Stamford Hospital, ${ }^{\mathrm{c}}$ Stamford, Conn; Upstate University Hospital, ${ }^{\mathrm{d}}$ Syracuse, NY; Caromont Health, ${ }^{\mathrm{e}}$ Gastonia, NC; Arnot Ogden Medical Center, ${ }^{\mathrm{f}}$ Elmira, NY; Methodist LeBonheur Healthcare, ${ }^{\mathrm{g}}$ Germantown, Tenn; Bassett Medical Center, ${ }^{\mathrm{h}}$ Cooperstown, NY; St Francis Hospital, ${ }^{\mathrm{i}}$ Greenville, SC; and The Valley Hospital, ${ }^{\mathrm{j}}$ Ridgewood, NJ.

Disclosures: Alex Zapolansky reports lecture fees from CorMatrix and equity ownership in Atricure. The other authors have nothing to disclose with regard to commercial support.

Read at the 94th Annual Meeting of The American Association for Thoracic Surgery, Toronto, Ontario, Canada, April 26-30, 2014.

Received for publication April 18, 2014; revisions received July 9, 2014; accepted for publication Aug 18, 2014; available ahead of print Oct 5, 2014.

Address for reprints: Robert Lancey, MD, Cardiovascular and Thoracic Services, Maryview Medical Center, 3636 High St, Portsmouth, VA 23707 (E-mail: Robert_lancey@bshsi.org).

0022-5223/ $\$ 36.00$

Copyright (c) 2015 by The American Association for Thoracic Surgery

http://dx.doi.org/10.1016/j.jtcvs.2014.08.059
See related commentary on page 858

Supplemental material is available online.

With the shift in health care economics from volume-based purchasing to value-based purchasing, numerous initiatives by payers have focused on rewarding for care high in quality, safety, and service, and penalizing care that is not. In October 2012, the Centers for Medicare and Medicaid Services presented the Hospital Readmission Reduction 


\section{Abbreviations and Acronyms \\ $\mathrm{CABG}=$ coronary artery bypass grafting \\ IABP $=$ intra-aortic balloon pump \\ STS $=$ Society of Thoracic Surgeons}

Program, which codified financial penalties on hospitals with excessive rates of readmission within 30 days of discharge. ${ }^{1}$ Initially designed for medical conditions, recent iterations have targeted surgical procedures, with coronary artery bypass graft $(\mathrm{CABG})$ surgery being considered as a candidate.

Many studies have examined the risk factors and predictors for readmission following heart operations. However, the findings have been inconsistent and in some cases contradictory. There is potential value in a predictive model for readmissions that would enable focusing finite resources on those at highest risk. We have previously reported such a model for patients following CABG in a single institution. ${ }^{2}$

Along with the variety of clinical factors that have been identified as associated with readmission, variations in patient demographic characteristics, including socioeconomic status, are also emerging as important predictors. ${ }^{3}$ A predictive model based on clinical factors alone might then be at risk for losing adaptability across institutions if there are powerful, nonclinical predictors at play that vary widely across institutions.

We report an approach to identifying patients at risk for readmission following CABG surgery in a multicenter academically affiliated network of community hospitals.

\section{METHODS}

This study received institutional review board approval with waiver of patient consent for use of de-identified registry data.

\section{Data-Related Methods and Definitions}

Using a Society of Thoracic Surgeons (STS) compliant registry, data were collected on consecutive patients undergoing isolated CABG in an 11-hospital network from 2007 to 2011. Not all hospitals contributed data for the entire time period. Time from surgery to readmission was calculated as the date of readmission minus the date of surgery, and time from discharge to readmission was calculated as the date of readmission minus the date of discharge.

The binary outcome of interest was readmission to the hospital within 30 days of the surgical procedure. Records with missing values on the outcome variable were excluded $(n=68)$, as were patients who died without readmission within 30 days of surgery $(n=6)$. Patients who died within 30 days of surgery but were readmitted first were included in the study. Data on readmission were obtained by the hospital data managers for those patients who were discharged alive and had not been readmitted to the operative hospital by direct contact with the patient and/or family.

Predictor variables included preoperative, intraoperative, and postoperative factors, as well as demographic characteristics, insurance status, discharge location, and body mass index. Insurance status was classified as self-pay, Medicaid, Medicare, and third-party payers. Discharge location was classified as extended care/other hospital, nursing home, or home. All complications and risk factors were dichotomized as either present or absent, with missing values assigned as absent.

Among readmitted patients, reasons for readmission were categorized according to their relatedness to CABG: clearly, possibly, and unlikely. Reasons that were categorized as clearly procedure-related were acute vascular complication, infection (conduit harvest site), infection (deep sternum), infection (mediastinitis), pericardial effusion/tamponade, and arrhythmia/heart block. Reasons classified as possibly procedure-related were coronary artery dysfunction, myocardial infarction and/or recurrent angina, deep venous thrombosis or pulmonary embolism, permanent cerebrovascular accident or transient ischemic attack, and pneumonia or other respiratory complication. Reasons classified as unlikely to be directly procedure-related were anticoagulation complications, congestive heart failure, and renal failure.

\section{Analytic Methods}

Readmission rates were calculated by year and by hospital. Distributions of preoperative, intraoperative, and postoperative risk factors for all patients were summarized as frequencies and proportions (or means \pm standard deviation, as appropriate). Comparison of risk factors between all readmitted patients and those not readmitted was carried out using $\chi^{2}$ tests (for categorical variables) or the independent samples $t$ test (for continuous variables).

Independent predictors of readmission were identified by first splitting the dataset at random into a training dataset and a validation dataset. The training dataset was used for variable selection and development of the logistic regression equation to predict readmission. The validation dataset was then used to apply the equation to the remaining group of patients as a means to assess its ability to predict readmission as a clinical tool.

In the training dataset, variables were tested at the univariate level for their relationship to readmission. For categorical variables, univariate tests were carried out using the $\chi^{2}$ test. For continuous variables, univariate tests were carried out using the independent samples $t$ test.

Statistically significant variables at the univariate level were entered into a stepwise logistic regression model to predict readmission. Variables independently predictive of readmission $(P<.05)$ at the conclusion of the stepwise modeling process formed the final logistic regression model. All analyses comprising the construction of the final logistic model were adjusted for clustering by hospital.

The finalized logistic regression equation was applied to the validation dataset, and the probability of readmission was estimated for each record. Sensitivity and specificity were calculated at 30 levels of probability of readmission. A receiver-operator characteristic curve was constructed, with the area under the curve estimated using trapezoidal approximation.

A subanalysis of readmission was carried out for 2 large hospitals in the network. These hospitals were chosen specifically to have disparate geographic and patient characteristics. Logistic regression models were constructed according to the methods described above. Training and validation datasets were not employed for this analysis.

In an additional subanalysis of readmitted patients, comparison of time to readmission across the 3-level CABG relatedness was carried out using 1-way analysis of variance. The recorded reasons for readmission were divided into categories estimating the likelihood for the readmission to be directly related to the procedure itself, as noted above. Scheffe post-hoc tests were used for pairwise comparisons of the mean days to readmission in each of the 3 readmission groups.

To assess the relationship between readmission and crude mortality, adjusted mortality, and adjusted combined mortality/morbidity, Pearson correlation was used. Adjusted mortality was calculated using the average predicted risk of mortality (from the STS database) divided by the true mortality rate for each hospital. Combined risk-adjusted mortality/morbidity 
Readmissions by hospital: 2007 - 2011

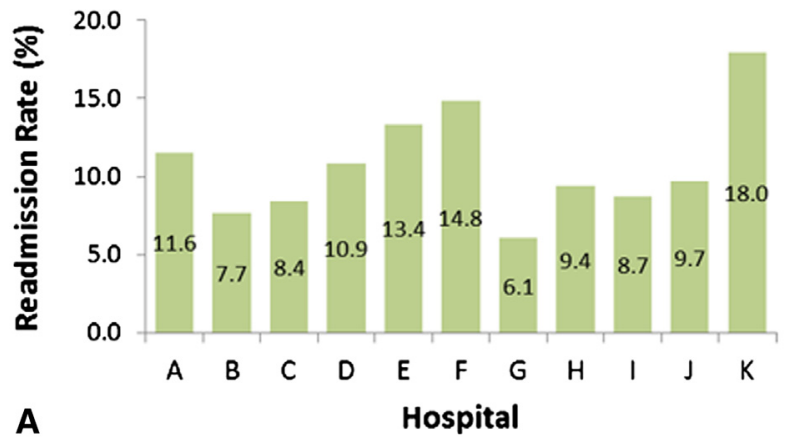

Readmissions by year: $2007-2011$

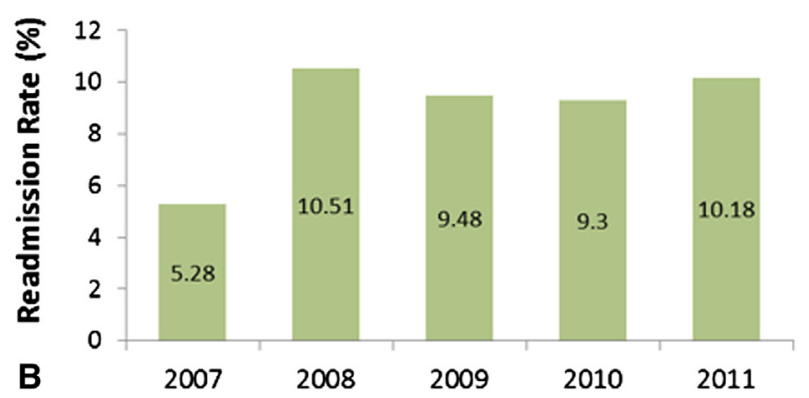

FIGURE 1. A, Readmission rates ( $\%$ ) by hospital during the study period (2007-2011). B, Readmission rates (\%) by year for the entire study cohort (2007-2011).

was calculated by dividing the STS predicted risk of mortality plus morbidity by the actual mortality/morbidity rates for each hospital. Morbidity was defined as any 1 of the following complications: prolonged ventilation, cerebrovascular accident, postoperative renal failure, deep sternal wound infection, and reoperation. All statistical analyses were performed with SAS (version 9.3; SAS Institute Inc, Cary, NC).

\section{RESULTS}

The readmission rate for the entire 4861 patient group was $9.2 \%$, with a range between hospitals of $6.1 \%$ to $18.0 \%$ (hospital mean, $10.9 \%$; median, $9.6 \%$; interquartile range, $8.4 \%-14.4 \%$ ) (Figure $1, A$ ), with no significant variation over time $(P=.24)$ (Figure $1, B)$. The training dataset consisted of 2341 patients of whom 207 $(8.8 \%)$ were readmitted, whereas the validation dataset consisted of 2520 patients of whom $240(9.5 \%)$ were readmitted.

The characteristics of the patient population as they relate to each of the preoperative, intraoperative, and postoperative variables was examined both for the entire population as well as for the groups that were and were not readmitted and are presented in Table 1. Significant differences were noted in the patients who were readmitted, including a higher proportion of self-pay patients and higher rates preoperatively of dyslipidemia, cerebrovascular disease, chronic obstructive pulmonary disease, diabetes, renal failure, previous coronary interventions, congestive heart failure, and myocardial infarction, as well as a higher body mass index. Readmitted patients were more likely to receive intraoperative blood products and less likely to receive an internal mammary artery graft, and postoperatively were more likely to be reintubated for respiratory distress, experience a leg wound infection, develop renal failure, receive blood products, or have a prolonged length of stay.

The significant independent risk factors for readmission within 30 days of surgery by logistic regression analysis are summarized in Table 2. The need for an intra-aortic balloon pump (IABP) appeared to be protective against readmission (odds ratio [OR], $0.40 ; 95 \%$ confidence interval [CI], $0.19-0.83 ; P=.015$ ), whereas the following were associated with increased odds for readmission: moderate chronic obstructive pulmonary disease (OR, 1.81; 95\% CI, 1.04-3.14; $P=.036$ ), history of cerebrovascular disease (OR, 1.56; 95\% CI, 1.09-2.24; $P=.016$ ), diabetes mellitus (OR, 1.44; 95\% CI, 1.08-1.93; $P=.014$ ), history of congestive heart failure (OR, 2.12; 95\% CI, 1.23-3.65; $P=.007$ ), IABP (OR, 0.40; 95\% CI, 0.19-0.83; $P=.015$ ), and use of intraoperative and/or postoperative blood products (OR, $1.76 ; 95 \%$ CI, $1.31-2.37 ; P=.0002)$.

Although the $c$ statistic for the training model $(\mathrm{n}=2341)$ was 0.643 , when applied to the validation dataset $(\mathrm{n}=2520)$, the area under the receiver-operator characteristic curve was reduced to 0.57 .

Separate logistic regression analyses of the factors independently associated with readmission at each of the 2 largest hospitals in the network revealed marked differences, both with each other as well as with the model generated for the entire network. Only higher body mass index (OR, 1.08; $95 \%$ CI, 1.04-1.12; $P=.0001$ ) was significantly associated with readmission at 1 hospital, whereas discharge to extended care (OR, 2.11; 95\% CI, 1.02-4.33; $P=.043)$ and renal failure (OR, 2.64; 95\% CI, 1.21-5.76; $P=.0149$ ) were the only independent risk factors at the other.

Most readmissions $(60.8 \%)$ occurred within 10 days of discharge with a mean time from surgery to readmission of $16.3 \pm 6.8$ days and a median of 16 days (interquartile range, 11-22 days) (Figure 2).

The plurality of readmissions $(44.5 \%)$ were categorized as clearly procedure-related, with $24.2 \%$ classified as possibly procedure-related and $31.3 \%$ considered unlikely to be directly procedure-related. When considering time to readmission, the mean number of days to readmission was significantly lower for clearly procedure-related (15.5 days) versus unlikely to be directly procedure-related (17.4 days; $P=.05$ ).

Across institutions, there is considerable variation in the pattern of readmissions (Figure 1) and the likelihood of relatedness to the surgical procedure (Figure 3). There was no correlation between readmission and either crude $(r=0.41 ; P=.20)$ or risk-adjusted mortality $(r=0.40$; $P=.23)$. There was however a correlation with the 
TABLE 1. Distribution of preoperative, intraoperative, and postoperative risk factors for readmission, for the entire sample and between readmitted versus nonreadmitted patients

\begin{tabular}{|c|c|c|c|c|}
\hline & $\frac{\text { Entire sample }}{(n=4861)}$ & $\frac{\text { Readmitted within } 30 \text { days }}{(n=447)}$ & $\frac{\text { Not readmitted }}{(n=4414)}$ & $P$ value \\
\hline \multicolumn{5}{|l|}{ Demographic characteristic } \\
\hline Male & 72.5 & 71.1 & 72.7 & .49 \\
\hline \multicolumn{5}{|l|}{ Race/ethnicity (not mutually exclusive) } \\
\hline Hispanic & 1.5 & 1.0 & 1.5 & .39 \\
\hline African American & 16.5 & 18.5 & 16.3 & .25 \\
\hline American Indian & 0.1 & 0 & 0.1 & .99 \\
\hline Asian & 1.2 & 1.5 & 1.2 & .67 \\
\hline Pacific Islander & 0.2 & 0 & 0.2 & .99 \\
\hline Other race & 1.2 & 1.5 & 1.1 & .48 \\
\hline White & 80.7 & 78.8 & 81.0 & .29 \\
\hline \multicolumn{5}{|l|}{ Insurance } \\
\hline Self-pay & 7.2 & 7.6 & 7.1 & .01 \\
\hline Medicaid & 35.3 & 27.9 & 36.1 & \\
\hline Government/Medicare & 61.0 & 57.5 & 50.3 & \\
\hline Third party & 6.6 & 7.1 & 6.5 & \\
\hline Age, y & $64.5 \pm 10.5$ & $65.2 \pm 10.9$ & $64.4 \pm 10.5$ & .14 \\
\hline \multicolumn{5}{|l|}{ Preoperative characteristic } \\
\hline Dyslipidemia & 74.1 & 78.3 & 73.7 & .04 \\
\hline Peripheral artery disease & 13.1 & 15.0 & 12.9 & .22 \\
\hline Cerebrovascular disease & 13.3 & 19.0 & 12.7 & .0002 \\
\hline \multicolumn{5}{|l|}{ Chronic obstructive pulmonary disease } \\
\hline None & 75.7 & 69.4 & 76.4 & .004 \\
\hline Mild & 13.8 & 16.3 & 13.6 & \\
\hline Moderate & 5.7 & 6.9 & 5.6 & \\
\hline Severe & 4.7 & 7.4 & 4.5 & \\
\hline Diabetes & 39.9 & 47.4 & 39.1 & .0006 \\
\hline Hypertension & 84.4 & 89.0 & 84.0 & .005 \\
\hline Renal failure & 3.4 & 5.2 & 3.2 & .03 \\
\hline Previous intervention & 28.1 & 34.0 & 27.5 & .003 \\
\hline Congestive heart failure (current) & 5.8 & 10.1 & 5.3 & $<.0001$ \\
\hline Congestive heart failure (past) & 4.1 & 7.6 & 3.7 & $<.0001$ \\
\hline Previous myocardial infarction & 42.9 & 48.4 & 42.4 & .02 \\
\hline Body mass index & $29.9 \pm 6.2$ & $30.7 \pm 7.2$ & $29.9 \pm 6.1$ & .03 \\
\hline \multicolumn{5}{|l|}{ Intraoperative characteristic } \\
\hline Ejection fraction calculated & 98.1 & 96.6 & 98.2 & .02 \\
\hline Intra-aortic balloon pump & 8.2 & 8.0 & 8.2 & .94 \\
\hline Intraoperative blood products & 25.1 & 32.9 & 24.3 & $<.0001$ \\
\hline Internal mammary artery (any vs none) & 95.0 & 91.5 & 95.4 & .0003 \\
\hline Reintubated & 2.6 & 3.8 & 2.5 & .09 \\
\hline Ejection fraction & $51.1 \pm 12.3$ & $50.2 \pm 12.7$ & $51.2 \pm 12.3$ & .11 \\
\hline Perfusion time, $\min$ & $98.4 \pm 31.9$ & $102.0 \pm 34.8$ & $98.0 \pm 31.6$ & .06 \\
\hline Crossclamp time, $\min$ & $70.8 \pm 22.6$ & $70.6 \pm 23.6$ & $70.8 \pm 22.5$ & .88 \\
\hline Intraoperative blood product, red blood cell units & $1.7 \pm 1.6$ & $1.78 \pm 1.5$ & $1.7 \pm 1.6$ & 60 \\
\hline \multicolumn{5}{|l|}{ Postoperative characteristic } \\
\hline Leg infection & 0.4 & 1.6 & 0.2 & $<.0001$ \\
\hline Sternum infection & 0.3 & 0.7 & 0.2 & .11 \\
\hline Neurologic & 0.6 & 0.7 & 0.6 & .76 \\
\hline Bleed & 2.0 & 2.7 & 2.0 & .29 \\
\hline Prolonged ventilation & 8.4 & 10.7 & 8.1 & .06 \\
\hline Vent longer than $72 \mathrm{~h}$ & 2.0 & 1.6 & 2.0 & .51 \\
\hline Dialysis & 0.7 & 1.1 & 0.7 & .38 \\
\hline Renal failure & 2.5 & 4.7 & 2.3 & .002 \\
\hline Intensive care unit hours & $66.1 \pm 87.2$ & $70.6 \pm 67.1$ & $65.6 \pm 89.0$ & .15 \\
\hline
\end{tabular}


TABLE 1. Continued

\begin{tabular}{|c|c|c|c|c|}
\hline & $\frac{\text { Entire sample }}{(n=4861)}$ & $\frac{\text { Readmitted within } 30 \text { days }}{(n=447)}$ & $\frac{\text { Not readmitted }}{(n=4414)}$ & $P$ value \\
\hline Vent hours & $13.2 \pm 44.3$ & $12.9 \pm 25.7$ & $13.2 \pm 45.8$ & .81 \\
\hline Received blood products & 34.4 & 48.6 & 33.0 & $<.0001$ \\
\hline Blood product, red blood cell units & $2.2 \pm 1.9$ & $2.4 \pm 1.8$ & $2.2 \pm 1.9$ & .28 \\
\hline \multicolumn{5}{|l|}{ Discharge location } \\
\hline Extended care/other hospital & 14.8 & 18.8 & 14.4 & .0001 \\
\hline Nursing home & 3.9 & 6.7 & 3.7 & \\
\hline Home & 81.2 & 74.4 & 81.9 & \\
\hline \multicolumn{5}{|l|}{ Other characteristics } \\
\hline Length of stay admit to discharge & $9.4 \pm 7.1$ & $10.2 \pm 5.5$ & $9.3 \pm 7.2$ & .0009 \\
\hline Length of stay surgery to discharge & $6.9 \pm 5.8$ & $7.3 \pm 3.9$ & $6.9 \pm 6.0$ & .06 \\
\hline
\end{tabular}

Values are presented as $\%$ or mean \pm standard deviation.

combined outcome of risk-adjusted morbidity and mortality $(r=0.66 ; P=.03)$.

\section{CONCLUSIONS}

Hospital readmissions are believed to be an indicator of suboptimal patient care, and thus may represent an unwarranted health care expense. The wide variation in incidence is believed to represent a disparity in care, as well as a potential opportunity for quality improvement and cost reduction. For this reason, in 2012 the Centers for Medicare and Medicaid Services launched the federal Hospital Readmissions Reduction Program. Hospitals with higher-than-predicted readmission rates for 3 prespecified diagnoses had their total Medicare reimbursement for fiscal year 2013 cut by up to $1 \%$ based on calculations of risk. These payment cuts were made public, and increase to $2 \%$ in 2014 , with additional diagnoses added. ${ }^{1}$ Two-thirds of hospitals $(66.7 \%)$ were penalized during the first year with a similar percentage (although slightly different distribution) in the second year of the

TABLE 2. Risk factors associated with readmission within $\mathbf{3 0}$ days of coronary artery bypass graft surgery (training dataset $n=2341$ ) on multivariable logistic regression

\begin{tabular}{lcccc}
\hline \multicolumn{1}{c}{ Parameter } & Coefficient & $\begin{array}{c}\text { Odds } \\
\text { ratio }\end{array}$ & $\begin{array}{c}\mathbf{9 5} \% \text { Confidence } \\
\text { interval }\end{array}$ & $\begin{array}{c}\boldsymbol{P} \\
\text { value }\end{array}$ \\
\hline Intercept & -2.857 & & & \\
No COPD & Reference & & & \\
Mild COPD & -0.0209 & 0.98 & $0.64-1.51$ & .9242 \\
Moderate COPD & 0.5912 & 1.10 & $1.04-3.14$ & .0360 \\
Severe COPD & 0.4553 & 1.58 & $0.92-2.71$ & .0982 \\
CVD & 0.4451 & 1.56 & $1.09-2.24$ & .0160 \\
Diabetes mellitus & 0.3652 & 1.44 & $1.08-1.93$ & .0142 \\
History of CHF & 0.7525 & 2.12 & $1.23-3.65$ & .0066 \\
IABP & -0.9160 & 0.40 & $0.19-0.83$ & .0146 \\
Blood products & 0.5673 & 1.76 & $1.31-2.37$ & .0002 \\
$c$ Statistic $=0.643$ & & & & \\
\hline
\end{tabular}

$C O P D$, Chronic obstructive pulmonary disease; $C V D$, cerebrovascular disease; $C H F$, congestive heart failure; $I A B P$, intra-aortic balloon pump. program. Despite considerable interest and study within the medical profession, 2 questions regarding hospital readmissions remain largely unanswered: What are the actionable risk factors for readmission? and, How well is readmission correlated with other metrics of quality?

Risk models for readmission have had only limited predictive ability, with a $c$ statistic in the 0.56 to 0.72 range, ${ }^{4}$ with differing analyses of the same large national surgical registry (American College of Surgeons National Surgical Quality Improvement Program) arriving at markedly different conclusions. ${ }^{5,6}$ Coronary artery bypass surgery remains 1 of the most common and well-studied operations performed; yet risk factors for readmission following CABG surgery vary according to study, with careful analyses of several large and well-accepted cardiac surgical databases revealing differing risk factors and a disappointing discriminative $c$ statistic between 0.62 and 0.65 . $^{7-9}$ It is therefore not surprising that our risk model fell into a similar range.

Many of the risk factors-diabetes, congestive heart failure, chronic obstructive pulmonary disease, and blood transfusions-identified in this study have been noted in previous studies of patients undergoing cardiac surgery, ${ }^{7-10}$ although the negative predictive value of the use of IABP has not previously been noted. Whether this finding represents the value of aggressive surgical care or the competing factor of prolonged hospital length of stay as a negative predictor of 30-day postoperative readmission is not discernible for the data available. What emerges very clearly from our study is that the variability in the report of risk factors is not unique. In fact, when logistic regression analysis was independently applied to the 2 largest hospitals in our study, the risk factors that emerged were completely different between sites. Of note, 1 hospital is an inner-city hospital serving a lower socioeconomic population, whereas the other is a suburban hospital in a well-insured and relatively wealthy community.

There are many reasons for this variability in reported risk factors, as well as modest discriminative ability of the 


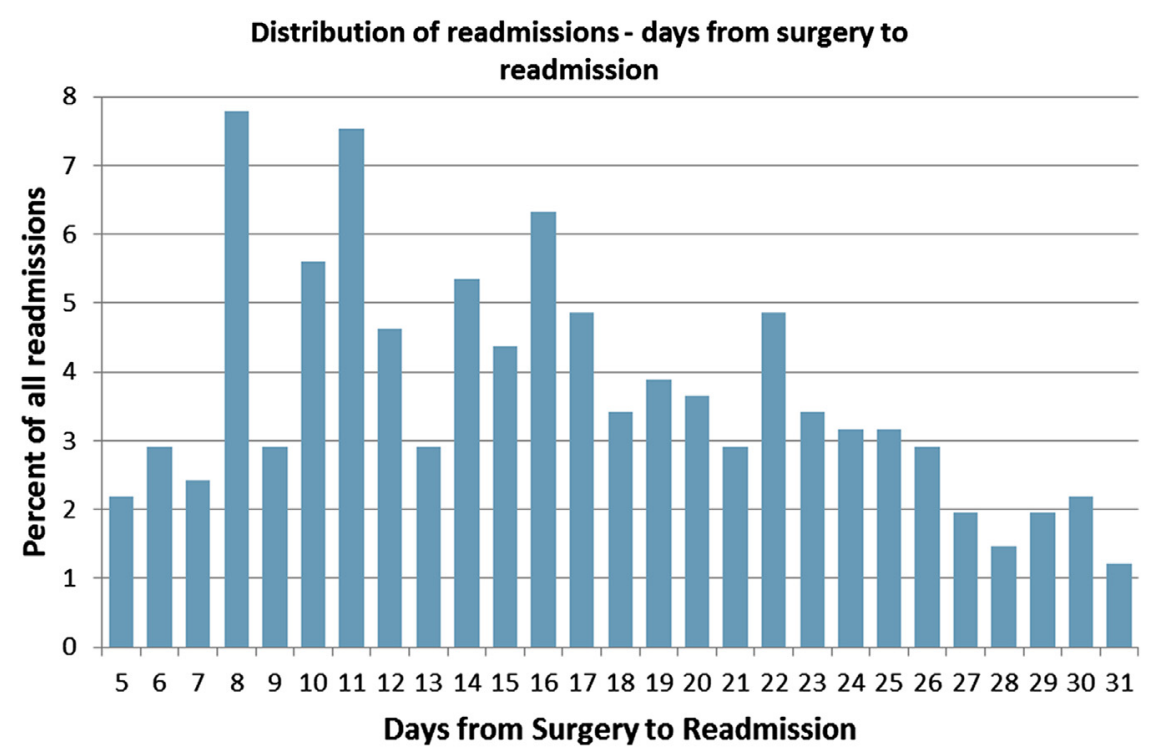

FIGURE 2. Distribution of readmissions by days from surgery to readmission.

risk models generated. First, death is a competing variable for readmission; yet many of the risk factors for mortality are the same as those for readmission. ${ }^{11}$ Therefore the ability to statistically distinguish the 2 is limited. Second, there are many factors not incorporated into clinical or administrative databases that may influence readmission.

Household income has been demonstrated to correlate with risk of readmission following $\mathrm{CABG},{ }^{12}$ other major operations, ${ }^{13}$ and in medical hospitalization. ${ }^{14}$ Nonetheless, the Centers for Medicare and Medicaid Services, in an effort not to bias care, has decided not to include socioeconomic status in their decisions regarding readmission penalties, resulting in a disproportionate share of the penalty falling upon safety net hospitals who care for a larger share of the poor. ${ }^{15}$ Multiple other factors may influence readmission after CABG surgery, such as multiple

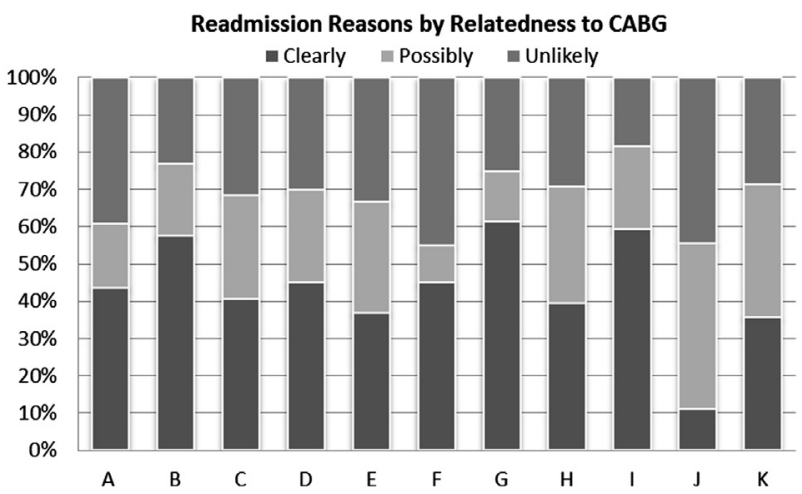

FIGURE 3. Percentage of readmissions that are clearly, possibly, and unlikely procedure-related to coronary artery bypass graft procedure, by hospital. $C A B G$, Coronary artery bypass grafting. prior emergency department visits, ${ }^{16}$ living alone,${ }^{17}$ social support, health beliefs, health care self-efficacy, and social norms. ${ }^{5,18}$ Indeed, differences in hospital quality and case mix have little effect on hospital readmission when compared with socioeconomic factors and community hospital bed and physician supply. ${ }^{3}$ Patient demographics, nursing home quality, and particularly access to care have also been found to play a more significant role in readmission rates. ${ }^{19}$

The second issue that our study addresses is whether or not readmission is an appropriate metric of quality, or rather a function of use. Our data did not demonstrate a significant correlation between mortality and readmission, consistent with finding in the California state registry, ${ }^{12}$ but somewhat at variance with the study of multiple surgical procedures using Medicare data. ${ }^{13}$ In New York State, interestingly enough, mortality for CABG surgery decreased over the same time period that readmission rates increased. ${ }^{7} \mathrm{We}$ found a correlation between the combined risk-adjusted outcome of mortality and complications, which is consistent with recent findings with patients undergoing noncardiac surgery in the American College of Surgeons National Surgical Quality Improvement Program project. ${ }^{6}$ Given the wide variability in readmission rates and the multiple factors that appear to contribute in different settings, it becomes increasingly challenging to use readmission rate as a metric of quality. Indeed, recent analysis of the Medicare beneficiaries undergoing CABG surgery found that $55 \%$ of the variation in readmissions rates was explained by measurement noise, with only $4 \%$ attributable to patient characteristics. The vast majority of hospitals were not able to achieve a minimum acceptable level of reliability for readmission rates. ${ }^{20}$ 
Given the complexity of the issue, the most productive area for surgeons to focus quality improvement efforts might be in the arena of potentially preventable readmissions. For certain conditions, readmissions may in fact be optimal medical care, and a measure of good rather than poor quality. ${ }^{21}$ However, our findings that nearly one-half of readmissions were very likely procedurerelated, and that these readmissions were more likely to occur shortly after discharge, may provide a subgroup on which to focus future care efforts-both in the prevention of surgical complications and in the prompt recognition and treatment of those that occur (Table E1). This may include follow-up with the surgical team within the first week after discharge, to allow more prompt recognition and treatment of those complications. Likewise, earlier recognition may be enhanced by fostering closer relationships and communication with health care providers who care for patients after discharge, including those in home health care and rehabilitation centers.

There are numerous limitations to our study. Although the STS dataset is robust, the accuracy of the data, especially regarding readmission rate, could not be corroborated with other sources and is thus vulnerable to variations in the diligence and accuracy of data collection across multiple sites. However, the generally high level of data management within this surgical network gives reasonable assurance of the integrity of the information on this parameter.

Second, the data available, as with most surgical databases, monitor readmission within 30 days of surgery, rather than within 30 days of discharge, as recorded by the Centers for Medicare and Medicaid Services. Therefore, prolonged hospital stay may be a competing variable that may bias the findings, underestimating the predictive value of complications or associated morbidities. It should be noted that with a mean length of stay of $6.93 \pm 5.83$ days, $95.3 \%$ of patients in our study had a postsurgical length of stay $\leq 14$ days. It is therefore not surprising that length of stay was a univariate predictor of readmission, and therefore unlikely to significantly bias our study results. Moreover, because the purpose of this work was to try to identify potentially modifiable or actionable risk factors for preventable readmissions, we believed that the source of data was the most appropriate.

Third, the lack of information regarding socioeconomic and hospital demographic factors limited the ability to more fully discriminate readmission risk in our model, although this finding has important clinical and social policy implications. Further, this study does not address returns to acute care that do not result in hospitalization, although this may represent a considerable proportion of the postdischarge need for acute care. ${ }^{22}$

Fourth, the criteria for relatedness to surgery may be inexact. On some level, all reasons for readmission, except for the most obscure, have some relationship to the index admission and the surgery performed. However those patients who have underlying problems; for example, with congestive heart failure, may be prone to readmission regardless of the operative care. Similarly, those patients with anticoagulation-related issues frequently reflect issues of follow-up management rather than surgical care per se.

Despite the limitations of the data, our analysis of CABG readmission data from a network of community hospitals that vary in size and demographic characteristics support the conclusions that there may be many nonclinical factors that influence readmission; readmission rates and associated risk factors may vary considerable from hospital to hospital; earlier readmissions tend to be more procedure-related and may provide an opportunity from targeted intervention, whereas later readmissions tend to be more patient-related; and therefore considerable caution should be exercised in attempting to apply uniform standards or strategies to address post-CABG readmission.

\section{References}

1. Department of Health and Human Services, Centers for Medicare \& Medicaid Services. Medicare program; hospital inpatient prospective payment systems for acute care hospitals and the long-term care hospital prospective payment system and fiscal year 2013 rates; hospitals' resident caps for graduate medical education payment purposes; quality reporting requirements for specific providers and for ambulatory surgical centers. 42 CFR Parts 412, 413, 424, and 476. Federal Register. 2012;77:53258-750

2. Currie KB, Lancey R. A predictive model for readmission within 30 days after coronary artery bypass grafting. J Am Coll Surg. 2011;213:S107.

3. Joynt K. Impact of community factors on readmission rates. Circ Cardiovasc Qual Outcomes. 2012;5:A12.

4. Kansagar D, Englander H, Salanitro A, Kagen D, Theobald C, Freeman M, et al. Risk prediction models for hospital readmission; a systemic review. JAMA. 2011; 306:1688-98.

5. Lucas DJ, Haider A, Haut E, Dodson R, Wolfgang CL, Ahuja N, et al. Assessing readmission after general, vascular, and thoracic surgery using ACS-NSQIP. Ann Surg. 2013;258:436-9.

6. Glance LG, Kellerman AL, Osler TM, Li Y, Mukamel DB, Lustik S, et al. Hospital readmission after non-cardiac surgery: the role of major complications. JAMA Surg. 2014;149:439-45.

7. Hannan EL, Zhong Y, Lahey SJ, Culliford AT, Gold JP, Smith CR, et al. 30-Day readmissions after coronary artery bypass graft surgery in New York State. J Am Coll Cardiol Interv. 2011;4:569-76.

8. Shahian DM, He X, O'Brien S, Grover FL, Jacobs JP, Edwards FH, et al. Development of a clinical registry-based 30-day readmission measure for coronary artery bypass grafting surgery. Circulation. 2014;130:399-409.

9. Brown JR, Parikh CR, Ross CS, Kramer RS, Magnus PC, Chaisson K, et al; for the Northern New England Cardiovascular Disease Study Group. Impact of perioperative acute kidney injury as a severity index for thirty-day readmission after cardiac surgery. Ann Thorac Surg. 2014;97:111-7.

10. D’Agostino RS, Jacobson J, Clarkson M, Svensson LG, Williamson C, Shahian DM. Readmission after cardiac operations: prevalence, patterns and predisposing factors. J Thorac Cardiovasc Surg. 1999;118:823-32.

11. Hannan EL, Racz MJ, Walford G, Ryan TJ, Isom O, Bennett E, et al. Predictors of readmission for complications of coronary artery bypass graft surgery. JAMA. 2003;290:773-80.

12. Li Z, Amstrong EJ, Parker JP, Danielsen B, Romano PS. Hospital variation in readmission after coronary artery bypass surgery in California. Circ Cardiovasc Qual Outcomes. 2012;5:729-37.

13. Tsai TC, Joynt KE, Orav EJ, Gawande AA, Jha AK. Variation in surgical readmission rates and quality of hospital care. N Engl J Med. 2013;369:1134-42.

14. Lindenauer PK, Lagu T, Rothberg MB, Avrunin J, Pekow PS, Wang Y, et al. Income inequality and 30 day outcomes after acute myocardial infarction, heart failure and pneumonia: retrospective cohort study. BMJ. 2013;346:f521. 
15. Joynt KE, Jha AK. Characteristics of hospitals receiving penalties under the Hospital Readmissions Reduction Program. JAMA. 2013;309:342-3.

16. Slamowicz R, Erbas B, Sundararajan V, Dharmage S. Predictors of readmission after elective coronary artery bypass graft surgery. Aust Health Rev. 2008;32: 677-83.

17. Murphy BM, Elliott PC, LeGrande MR, Higgins RO, Ernest CS, Goble AJ, et al. Living alone predicts 30-day hospital readmission after coronary artery bypass graft surgery. Eur J Cardiovasc Prev Rehabil. 2008;15:210-5.

18. Mitchell SE, Gardiner PM, Sadikova E, Martin JM, Jack BW, Hibbard JH, et al. Patient activation and 30-day post-discharge hospital utilization. J Gen Intern Med. . 2014;29:349-55.

19. Herrin J, St Andre J, Kenward K, Joshi MS, Audet AJ, Hines SC. Community factors and hospital readmission rates. Health Serv Res. April 9, 2014 [Epub ahead of print].

20. Shih T, Dimick JB. Reliability of readmission rates as a hospital quality measure in cardiac surgery. Ann Thorac Surg. 2014;97:1214-8.

21. Gorodeski EZ, Starling RC, Blackstone EH. Are all readmissions bad readmissions? N Engl J Med. 2010;363:297-8.

22. Fox JP, Suter LG, Wang K, Wang Y, Krumholz HM, Ross JS. Hospital-based, acute care use among patients within 30 days of discharge after coronary artery bypass surgery. Ann Thorac Surg. 2013;96:96-104.

\section{Discussion}

Dr Hersh S. Maniar (St Louis, Mo). The paper has several strengths, including a large number of patients undergoing a single procedure at multiple institutions. I think this offers you a unique opportunity because there were 11 institutions in the study. Why was there such a wide range of readmission rates amongst the 11 hospitals that participated in the study, since it was 1 procedure?

Dr Lancey. We found that there were marked similarities in clinical factors among the patients. But with the hospitals, again, not knowing the socioeconomic data but knowing the characteristics of the hospitals themselves and of the patients they serve, we suspected that there was greater influence from socioeconomic factors. So it gave us a socially heterogeneous group of patients, although clinically homogenous.

Dr Maniar. One of the things that we will all try to glean from this study is what readmissions are preventable. Seventy percent of your readmissions were deemed procedure-related; however, many may argue that nearly all readmissions are related to the procedure or due to a patient's cardiovascular disease. For example, how would a patient with perioperative atrial fibrillation who goes home taking warfarin and then presents with a bleeding complication be characterized? Is that related, unrelated, or possibly?

Dr Lancey. That's a very good question. That would be a clearly related. What we did was classify patients with infections, arrhythmias, and heart block, pericardial effusions and tamponade, and acute vascular complications as those that were clearly related. So this is a patient we would put into the clearly related category.

Dr Maniar. You have acknowledged the limitations of the manuscript, specifically with respect to the lack of socioeconomic variables included in the investigation. Given this sizable limitation, what can we do with this data and how does it affect or change your practice?

Dr Lancey. That's a good question. The next step in this analysis would be to gain a better understanding of the socioeconomic status and patient demographics in a larger study.
Clearly these factors play a greater role than has been previously recognized, as a number of recent studies have shown.

Dr Harold L. Lazar (Boston, Mass). Were you able to correlate with any of those hospitals that had a fast-track protocol whether there is any influence on those patients who met that protocol and were discharged? Was there any relationship between their rate of return versus people who were in hospitals that didn't have that?

Dr Lancey. No, we didn't, but that's a good thought.

Dr Hossein Almassi (Milwaukee, Wis). It appears that socioeconomics was a major part for this patient to be readmitted. Is it related to their preexisting condition or to the attempts on part of the hospital to discharge them out of the hospital so that it cuts down the cost for the hospital because these are maybe nonpaying patients? Or are some other issues involved and that results in patients coming back because they were discharged before they really were optimized or the lack of support after hospital discharge?

There appears to be multiple things and factors involved that make your admission for these patients who are self-pay. Under that terminology, I gather it means socioeconomically disadvantaged also. Can you elaborate on that, please?

Dr Lancey. Sure. We intentionally did not use self-pay as a replacement for lower socioeconomic status. I think it's a poor surrogate for that.

But access to care is a variable that we could hopefully quantify in the future to determine its role. That's why we list as 1 of the limitations the fact that a number of patients may have gone back for acute care but not rehospitalization. For instance, you can use observation status for less than 24 hours, and avoid the label of readmission.

So it's not so much gaming the system, but there are ways to avoid the readmission label on patients, and that practice I think is more hospital-specific.

Dr Thomas J. Vander Salm (Salem, Mass). Are readmissions a priori bad? I ask that because I've come to the conclusion that we sometimes delay or defer readmission when an earlier readmission will sometimes advantage the patient.

Dr Lancey. It depends on whose perspective. For patients, readmissions are generally necessary and therefore good. But for Medicare, because of the payment ramifications, I think they're universally seen as bad.

Dr Paul Kurlansky (New York, $N Y$ ). Readmission is really a measure of use. It's not a measure of quality. And the problem is that the payers, because it's a financial issue, are confusing the 2 .

In this study, we looked at correlation between readmission and mortality; for example, and there was no correlation. So whether or not it is even a viable metric for quality is extremely questionable at best, and it depends partially on the disease process you're looking at.

There's a nice letter that Gene Blackstone wrote to the New England Journal of Medicine where he showed that there is an inverse correlation between mortality and readmission in congestive heart failure because death is a competing variable.

So it may be that readmission in that situation can be a sign of good care rather than bad care, and so the readmission rate of zero is not necessarily a good thing. 
TABLE E1. Factors most clearly associated with 30-day readmission following coronary artery bypass surgery

\begin{tabular}{lcc}
\hline \multicolumn{1}{c}{ Reason for readmission } & $\begin{array}{c}\text { Number of } \\
\text { readmissions } \\
(\mathbf{n}=\mathbf{4 4 7})\end{array}$ & $\begin{array}{c}\text { Percent of all } \\
\text { readmissions }\end{array}$ \\
\hline Acute vascular complication & 8 & 1.79 \\
Anticoagulation complication - & 5 & 1.12 \\
$\quad$ pharmacologic & & \\
Arrhythmia/heart block & 40 & 8.95 \\
Congestive heart failure & 55 & 12.3 \\
Coronary artery dysfunction & 3 & 0.67 \\
Deep venous thrombosis & 1 & 0.22 \\
Infection - conduit harvest site & 18 & 4.03 \\
Infection - deep sternum & 13 & 2.91 \\
Myocardial infarction and/or & 22 & 4.92 \\
$\quad$ recurrent angina & & \\
Pulmonary embolism & 2 & 0.45 \\
Pericardial effusion and/or tamponade & 10 & 2.24 \\
Permanent cerebrovascular accident & 5 & 1.12 \\
Pneumonia or other respiratory & 70 & 15.66 \\
$\quad$ complication & & \\
Renal failure & 3 & 0.67 \\
Transient ischemic attack & 5 & 1.12 \\
Other - nonrelated readmission & 77 & 17.23 \\
Other - related readmission & 110 & 24.61 \\
\hline
\end{tabular}

